\title{
Public et représentations dramatiques dans le nord de la France à la fin du Moyen Âge
}

\author{
KATELL LAVÉANT
}

S i le public est la raison d'être d'une représentation dramatique, la critique n'a pas toujours pu étudier en détail sa nature, ses réactions face à une pièce, sa participation lors d'une représentation. En effet, nous manquons de données le concernant, et nous en sommes souvent réduits aux suppositions. De plus, étant donné la multiplicité des types de représentations et de manifestations dramatiques aux $\mathrm{XV}^{\mathrm{e}}$ et $\mathrm{XVI} \mathrm{I}^{\mathrm{e}}$ siècles, il est peu aisé d'avoir une vue d'ensemble sur ce public, quand le champ de la recherche se porte en général sur un type de pièce spécifique, ${ }^{1}$ et qu'on peut d'autre part se demander si le public, suivant les différentes manifestations dramatiques possibles, est toujours le même.

D'ailleurs, faut-il bien parler d'un public unique, rassemblant nobles, bourgeois et peuple, quand les descriptions des pièces jouées lors d'entrées royales, pour ne citer qu'elles, attestent de la complexité conceptuelle des scènes, et laissent envisager la possibilité qu'une grande partie du public, la moins éduquée, ne comprennent pas grand-chose à ce qui est mis en scène devant elle? Ne faut-il pas plutôt envisager l'existence d'un public fractionné, de plusieurs publics dans la masse des spectateurs d'une manifestation théâtrale, dont l'intérêt et le degré de compréhension de la représentation qui s'offre à eux varient selon ce qui est mis en scène, de la farce qui réjouit tout le monde, aux scènes allégoriques complexes disposées sur le parcours du prince qui fait son entrée dans une ville? Et n'y aurait-il pas alors d'autres facteurs de différenciation des publics?

C'est à partir de ces questions que j'aimerais apporter des éléments de réponse concernant le public des représentations à caractère théâtral à la fin du Moyen Âge dans le nord de la France. J'aimerais tenter de donner une vue d'ensemble qui

${ }^{1} \mathrm{Si}$ de nombreuses études existent sur les mystères ou les farces, pour ne citer que ces types de pièces, il est rare que des études d'ensemble s'attachent à étudier tous les types de pièces existant dans une région ou à une époque précises. 
englobe tous les types de représentations pour lesquelles on a des informations concernant la composition, les réactions ou la participation du public dans cette région. Cela m'amènera à considérer donc aussi bien le public des farces que celui des mystères, en passant par celui des tableaux vivants présentés sur le parcours des processions religieuses ou des entrés royales, et à prendre en compte non seulement les données d'archives et les descriptions faites par des contemporains, mais aussi les renseignements que peut nous apporter l'iconographie, qui, bien que rare et déjà amplement étudiée, me semble ici d'autant plus pertinente qu'elle est le plus souvent issue de régions liées à celle que je me propose d'étudier.

Étant donné l'ampleur d'un tel programme, je ne peux espérer mener à bien ici une étude exhaustive et définitive; mais $\mathrm{j}$ 'espère pouvoir apporter un éclairage nouveau à la réflexion d'ensemble à partir du cas d'une région géographique cohérente, notamment à partir de pièces d'archives partiellement inédites ou restées consignées aux ouvrages des érudits du XIX ${ }^{\mathrm{e}}$ siècle.

Mon étude porte essentiellement sur les villes du nord de la France et du sud de la Belgique actuelle, dans une zone géographique qui s'étendrait d'Abbeville à Valenciennes et d'Amiens à Tournai. Il apparaît en effet, quand on étudie plus précisément les données d'archives concernant les villes de cette région, qu'il existe entre ces dernières des liens reposant sur l'organisation et la participation mutuelles à des concours dramatiques et des fêtes locales dont la représentation de pièces jouées par des compagnies venant des villes voisines est une part essentielle. Revenant sur les propos d'Henri Pirenne à propos des rivalités existant à la fin du Moyen Âge entre les villes de la région et de leur tentation de s'isoler de leurs voisines, Alan Knight souligne qu'il faut relativiser cette vision négative à la lumière des données concernant l'implication de ces villes dans l'activité théâtrale: 'The records of dramatic activities in the provinces of Flanders and Picardy place this hostility in an altogether different light. They reveal a coexisting spirit of friendly rivalry and interurban cooperation that tended to counterbalance the negative forces. ${ }^{2}$

Et de fait, l'on s'aperçoit, notamment à la lecture des registres de comptes de ces différentes villes, que ces derniers mentionnent extrêmement fréquemment la venue de troupes d'acteurs des villes voisines pour participer à un concours dramatique, prendre part à une fête qui réunit des troupes de toute la région ou simplement jouer une pièce hors de toute manifestation dramatique; et les troupes de cette même ville se déplacent elles-mêmes à l'extérieur, pour les mêmes raisons que les troupes qui sont venues dans la ville.

Prenons le cas d'Arras: cette ville occupe une position importante dans les échanges dramatiques interurbains dans le nord de la France, sans doute du fait de sa position géographique centrale, qui permet aux troupes de toutes les villes alentour

2 A. E. Knight, 'Drama and Society in Late Medieval Flanders and Picardy', Chaucer Review, 14 (1980), 379-89 (p. 380), citant H. Pirenne, Les anciennes démocraties des PaysBas (Paris: Flammarion, 1910). 
de se rencontrer sans avoir à se déplacer sur de trop grandes distances. Les registres de compte ont été dépouillés et classés par thèmes au XIX ${ }^{\mathrm{e}}$ siècle par Adolphe Guesnon: son fichier 'jeux scéniques" ${ }^{3}$ nous apporte de précieux renseignements sur l'activité d'Arras aux XV et $\mathrm{XVI}^{\mathrm{e}}$ siècles. La ville subventionne et confie l'organisation de la plupart des manifestations dramatiques à un Abbé de Liesse, qui dirige la confrérie joyeuse du même nom et est élu chaque année par ses confrères. Il est notamment chargé d'organiser la fête du Gras Dimanche (le dernier dimanche avant le début du Carême) qui a lieu chaque année, ainsi que le confirme cet extrait de compte: pour l'année de comptes 1454-55, Pierre de Hennin, Abbé de Liesse de l'année en cours, reçoit huit livres 'pour avoir recu le jour de gras dimanche plusieurs compagnons de plusieurs villes qui y sont venus jouer jeux de personnages ainsi qu'il est accoustumé faire chascun an'. Les noms de ces villes sont précisés en 1494 et 1497: il s'agit de Cambrai, Douai, Lille, Béthune, Saint-Pol et d'autres villes voisines (le compte de 1490 précise qu'il s'agit de villes d'Artois). En outre, des rencontres plus ponctuelles ont lieu sous la forme de concours dramatiques: en 1431, le concours réunit à Arras les villes de Cambrai, Douai, Valenciennes, Hesdin, Montreuil, Saint-Quentin. ${ }^{4}$ Outre l'organisation de diverses manifestations dramatiques à domicile, Arras envoie également l'Abbé de Liesse et ses compagnons la représenter lors des fêtes annuelles des villes voisines. Ainsi, on retrouve l'Abbé de Liesse à Cambrai pour la fête annuelle du Vingtième, ${ }^{5}$ où il retrouve des joueurs d'Amiens et de Saint-Quentin (de nombreuses dates sont citées dans les comptes du début du XV siècle jusqu'à 1509 au moins), à L'Écluse en 1441 pour un concours de rhétorique, à

${ }^{3}$ Consistant en fiches manuscrites et classées par thèmes (celui qui nous intéresse est nommé 'jeux scéniques, mystères, confréries littéraires') ce fichier établi en 1885 et conservé à la Bibliothèque Municipale d'Arras fait un relevé précis et détaillé des registres de comptes d'Arras de la fin du XIV à la fin du XVI ${ }^{\mathrm{e}}$ siècle.

${ }^{4}$ Fichier Guesnon: en septembre 1431, l'Abbé de Liesse, Andrieu Hanebot, organise un concours de rhétorique et de jeux, selon les catégories suivantes:

- celui qui saura le mieux démontrer pourquoi paix ne vient en France, prix d'argent de 8 onces (obtenu par ceux de Hesdin); deuxième prix: agneau d'argent de 5 onces (Cambrai)

- celui qui saura le mieux faire le sage en ivrogne, un pot d'argent (Cambrai), et le deuxième: un voire d'argent (Hesdin)

- celui qui allumera le mieux à peu de frais un falot d'argent (St-Quentin)

- celui qui prononcera le mieux, une couronne d'argent (Douai)

- celui qui continuera le plus en jeux de vêpres un ours d'argent (Valenciennes)

- à la plus lointaine ville: une rose d'argent (Montreuil)

- au mieux chantant: une alouette d'argent (Cambrai)

- à la plus belle compagnie et chacun qui joua un agnelet d'argent.

Voir également Carlos Antonio La Serna Santander, Mémoire historique sur la bibliothèque dite de Bourgogne présentement bibliothèque publique de Bruxelles (Bruxelles: A. J. D. de Braeckenier, 1809), p. 165.

${ }^{5}$ La fête du Vingtième a lieu vingt jours après Noël. 
Béthune pour la fête de Jeunesse en 1463, à Douai pour le Jour de l'An en 1490, à Lille pour la fête du roi des Fous en 1497, pour ne citer que ces quelques mentions dans les registres de comptes.

On pourrait multiplier les exemples pour chaque ville de la région, qui tendent à prouver l'existence de réseaux de sociabilité interurbains fondés sur les échanges dramatiques entre villes, soit de façon ponctuelle quand une troupe d'une ville se déplace dans une ville voisine pour jouer une pièce, soit quand plusieurs villes se retrouvent dans une autre pour une manifestation dramatique qui peut durer plusieurs jours (ainsi la fête du Vingtième à Cambrai en 1490 dure trois jours).

De plus, la cohérence de cette région se trouve confirmée par sa situation géopolitique: toutes les villes considérées se trouvent incluses dans le duché de Bourgogne selon ses limites après le traité d'Arras de 1435, et restent dans cette zone d'influence pendant la période qui nous intéresse. Enfin, il faut tenir compte du facteur d'unité linguistique, qui explique que des villes comme Tournai et Mons ont davantage de liens culturels avec leurs voisines françaises qu'avec leurs voisines flamandes.

Il me semble donc que cette région relativement vaste, où la circulation des pièces et des acteurs est importante et où l'activité dramatique est particulièrement florissante aux $\mathrm{XV}^{\mathrm{e}}$ et $\mathrm{XVI}^{\mathrm{e}}$ siècles, présente un intérêt majeur pour une étude du public, notamment puisqu'elle permet de tirer des conclusions qui concernent une part notable du théâtre de la fin du Moyen Âge en France. De fait on y rencontre, pour la période, tous les types de représentations dramatiques possibles, sur lesquels il serait bon de s'attarder avant d'en venir à l'étude du public proprement dite, dans la mesure où ces différentes sortes de pièces peuvent justement induire l'existence d'un public différent. Si l'on cite habituellement les quatre types de pièces: mystère, moralité, farce et sottie, dont on trouve aussi des exemples, au moins sous la forme de mentions d'archives, dans le nord de la France, il me semble que si l'on veut mieux cerner le public des activités dramatiques, il faut ne pas s'en tenir à ces pièces exclusivement. Car si l'on rencontre effectivement sous ces termes un certain nombre de pièces attestées dans la région, on constate également l'existence d'autres types d'activité dramatique moins bien définis, mais qui peuvent rassembler le(s) même(s) public(s) que les précédents. En effet, pour reprendre l'exemple d'Arras, on s'aperçoit qu'il existe une multitude de représentations dramatiques dont il n'est pas toujours facile de déterminer la nature exacte. Tout d'abord, les pièces ne sont pas si clairement définies dans les archives. Ainsi, pour Saint-Omer, on répertorie les termes suivants dans les archives: 'mistère', utilisé très souvent pour désigner des moralités, 'jeu de folie', 'exemple'; et, encore plus indéterminé: 'histoire', 'ébattement', 'joyeuseté', 'jeux sur cars'. De même, on joue à Arras en 1461 des 'folies moralisées' pour fêter la fin des procès de vauderie qui se sont tenus dans la ville, ${ }^{6}$ sans que l'on puisse

${ }^{6}$ J. Huizinga, Le déclin du Moyen Âge (Paris: Payot, 1932), cité par J. Britte-Ashford, 'Le théâtre populaire en Bourgogne au Xv $v^{\mathrm{e}}$ siècle' (thèse de doctorat non publiée, Brigham Young University, 1972). 
déterminer ce que cette appellation désigne, et le plus souvent, quelle que soit la ville, les pièces ne sont désignées que sous le terme générique de 'jeux de personnages', sur le contenu desquels il faudrait s'interroger.

D'autre part, certains événements et fêtes religieuses donnent lieu à des mises en scène à caractère dramatique, elles aussi difficiles à définir. Les processions religieuses sont par exemple l'occasion d'émailler le parcours des fidèles de scènes bibliques: selon Louandre, en 1488, à Abbeville, 'on montre au peuple sur le marché les mystères de Jonas et du Vieux Testament et Nouveau Testament, pendant les processions générales faites pour préserver les habitants de la peste qui régnait autour de la ville, et demander au ciel une abondante moisson'; ' de même, à Arras en 1494, le roi des Sots et le duc du Lacq jouent à la procession 'plusieurs histoires du mistère de la Passion et de la Résurrection'. ${ }^{8}$ En quoi consistent exactement ces représentations? On comprend que le terme 'mystère' désigne ici non pas de longues pièces faisant appel à un grand nombre d'acteurs, mais de petites pièces courtes, sortes d'extraits ou d'épisodes tirés de la Bible et prenant un sens indépendamment de la plus longue histoire à laquelle elles appartiennent, comme l'indiquent par exemple la désignation de Jonas comme une histoire à part et l'expression 'plusieurs histoires du mistère'. D'ailleurs, il est fort probable que ces scènes aient souvent été muettes et mimées, voire consistant en de simples tableaux vivants, afin de ne pas perturber le bon déroulement de la procession et de laisser les fidèles se concentrer sur le message religieux plutôt que sur l'attrait de la dramatisation. De fait, en 1484 à Saint-Omer, on attribue quatre livres 'aux compagnons qui jouèrent à la procession par signes et après dîner par personnages la création du monde le jour du sacrement'. 'Le terme 'par signes' indique clairement qu'il s'agit pendant la procession d'un spectacle seulement mimé, mais il est intéressant de constater qu'une fois les dévotions achevées, le soir, on prend plaisir à assister de nouveau aux mêmes scènes, cette fois proprement jouées 'par personnages'.

Il en va de même lors des entrées dans les villes de personnages importants, qui sont autant d'occasions de mettre en place le long du parcours des scènes représentant des scènes bibliques, allégoriques ou mythologiques au XVI ${ }^{\mathrm{e}}$ siècle et d'offrir ainsi aux habitants de la ville ainsi qu'au prince et à sa suite des divertissements dramatiques. ${ }^{10}$ Là encore, la question se pose de savoir dans quelle mesure ces

${ }^{7}$ F. C. Louandre, Histoire d'Abbeville et du comté de Ponthieu jusqu'en 1789 (Paris: Joubert, Labitte, 1845), p. 319.

${ }^{8}$ Fichier Guesnon, ‘jeux scéniques'.

${ }^{9}$ Ibid. Guesnon n'indique pas si cet extrait est tiré des archives de Saint-Omer ou d'Arras. Cependant, il est probable que cet extrait figure dans le fichier parce que les joueurs qui ont joué ces histoires à la procession de Saint-Omer seraient originaires d'Arras.

${ }^{10}$ Sur les entrées royales, consulter B. Guenée et F. Lehoux, Les Entrées royales françaises de 1328 à 1515 (Paris: Centre National de la Recherche Scientifique, 1968), ainsi que C. de Mérindol, 'Théâtre et politique à la fin du Moyen Âge. Les entrées royales et autres cérémonies: mises au point et nouveaux aperçus', dans Théâtre et spectacles hier et aujourd'hui, 
scènes sont silencieuses ou non, figées ou mimées. Il semble que tous les cas de figure existent, sans doute en fonction de critères matériels (selon le temps que l'on pense devoir accorder à l'entrée, les souhaits éventuels du prince ou encore la présence ou non dans la ville de personnes capables de composer le texte des scènes). Ainsi, à Douai en 1439, pour l'entrée de Marguerite d'York et de Marie de Bourgogne, on joue plusieurs scènes religieuses: le Jugement de Salomon, l'Histoire d'Esther, l'Annonciation de Notre Dame, etc. L'entrée ayant lieu le soir, on allume de nombreux flambeaux pour éclairer les scènes, et selon le chroniqueur 'aussi il estoit bien besoing: car pluiseurs desdictes histoires estoient grandes et longhes et à pluiseurs personnages tout faisant par signe sans parler'. ${ }^{11}$ Mais à Abbeville en 1493, pour l'entrée de Charles VIII, on joue à l'entrée de la ville un véritable dialogue entre plusieurs allégories: Chief souverain, Abbeville, Bon Désir, Jocundité et Humble Service, qui vise à donner au roi la signification des tableaux sans doute muets situés sur le parcours qu'il va emprunter dans la ville. ${ }^{12}$

Avant d'en venir à la question de la différenciation des publics, il me semble qu'il faut s'arrêter un instant sur l'iconographie, dans la mesure où elle peut nous aider à appréhender les conditions matérielles dans lesquelles se trouve le public lors d'une représentation. On a coutume de dire que les témoignages iconographiques sur le théâtre médiéval sont rares. S'il est vrai que leur nombre est effectivement décevant par rapport à la proportion relativement importante des pièces conservées (ainsi que de celles pour lesquelles on ne possède que quelques renseignements épars: titre, lieu et/ou date de la représentation, noms des organisateurs et/ou des acteurs, etc.), il n'en reste pas moins qu'il permet d'établir une liste intéressante. De fait, on dispose d'un certain nombre de tableaux ou de gravures représentant différents types de représentations dramatiques, incluant ou non le public. Il faut noter cependant qu'en France, les références à l'iconographie se limitent le plus souvent à quelques documents, toujours les mêmes: la fameuse représentation par Fouquet du martyre de sainte Apolline, une peinture figurant dans un manuscrit de la Bibliothèque Municipale de Cambrai et représentant une pièce, vraisemblablement une farce, jouée sur de hauts tréteaux, ${ }^{13}$

Moyen Âge et Renaissance: Actes du $115^{e}$ congrès national des sociétés savantes, Avignon, 1990 (Paris: Éditions du comité de travaux historiques et scientifiques, 1991), pp. 179-212.

${ }^{11}$ F. Brassart, 'Relation officielle de la joyeuse entrée de Marguerite d'York, duchesse de Bourgogne et de sa belle-fille Marie de Bourgogne, le 19 novembre 1470, à Douai', Souvenirs de la Flandre-Wallonne: recherches historiques et choix de documents relatifs à Douai et la Province, 6.2 (1886), 155-66, qui cite le récit contenu dans les archives municipales de Douai (AA 85 , ancien $\mathrm{R}$, fol. $106^{\mathrm{V}}$ ).

${ }^{12}$ Consulter à ce propos A. Ledieu, 'Première entrée de Charles VIII à Abbeville', Bulletin Archéologique du Comité des travaux historiques, 1888, pp. 55-65.

${ }^{13}$ Cambrai, Bibiothèque Municipale, MS 126, recueil de chants religieux et profanes datant de 1542 . 
une autre de Pierre Balten: la Kermesse villageoise, ${ }^{14}$ et ce qui semble être le décor de la Passion de Valenciennes jouée en 1547, représenté par Hubert Cailleaux dans le MS. Rothschild I.7.3 de la Bibliothèque Nationale de France, constituent les principaux témoins cités lors de la discussion sur l'iconographie. Ces images ont suscité beaucoup de commentaires, notamment pour déterminer leur degré de réalisme concernant la représentation du dispositif scénique et du public. D'autre part, Willem Hummelen a produit au cours de ses travaux sur les rederijkers néerlandais de nombreux documents iconographiques dont la plupart est peu connue en France. ${ }^{15}$ Certes, on peut à juste titre se demander dans quelle mesure ces images de concours de rhétorique ou de kermesses villageoises peuvent aider également à l'étude du théâtre médiéval en France, du fait de leur région d'origine comme du fait qu'elles apparaissent tardivement au $\mathrm{XVI}^{\mathrm{e}}$ siècle. Cependant, il me semble qu'il est légitime de s'interroger sur leur intérêt en ce qui concerne les conditions de jeu et la position du public dans le nord de la France.

De fait, les villes du Nord ont été en contact avec leurs voisines du sud des PaysBas, aussi bien dans le domaine du commerce que de celui des échanges dramatiques. En effet, au-delà du cercle régional dans lequel les échanges dramatiques interurbains s'organisaient, on constate l'existence de liens entre ces villes françaises et les villes flamandes. Les villes du Nord sont en effet en contact avec ces dernières grâce aux concours dramatiques ou aux processions au cours desquelles les membres des compagnies joyeuses, des chambres de rhétorique ou des puys peuvent les rencontrer. Ainsi, Tournai invite régulièrement un grand nombre de villes voisines à participer à sa procession annuelle, au cours de laquelle sont joués des 'jeux sur cars', selon les comptes de la ville: en 1404, on paie 100 sous

audit Jehan de le Rue, pour avoir sur commandement des dessus dis Consaulx, porté lettres du sauf conduit de le Franque Feste de la Procession de la dite ville, dairainement passée, es ville d'Audenarde, Grantmont [Grammont], Alost, Tenremonde [Termonde], Anwiers [Anvers], Malisnes, Diestres [Diest], Louvaing, Brouxelle, Mons en Henau, Mauboege, Valenchiennes et Orchies, en le manière accoustumée. ${ }^{16}$

Selon Cauchie, des documents de la fin $\mathrm{du} \mathrm{XV} \mathrm{XV}^{\mathrm{e}}$ et du début du XVI ${ }^{\mathrm{e}}$ siècle nous apprennent que l'on annonçait la fête non seulement dans les villes citées mais également à Gand, Courtrai, Poperinghe, Dixmude, Messines, Thourout, Bruges, Ardenbourg, L'Écluse, Nivelles, Arras, Douai, Béthune, Lille, Aire, Ledain, Bailleul

${ }^{14} \mathrm{XVI}^{\mathrm{e}}$ siècle, original perdu, cinq copies sont conservées en différents endroits, dont le musée du théâtre d'Amsterdam.

${ }^{15}$ Pour ne citer qu'un article, Hummelen étudie de nombreux documents dans 'Toneel op de kermis, van Bruegel tot Bredero', Oud Holland, 103 (1989), 1-45 (consultable sur internet: http://www.dbnl.org/tekst/humm001tone01/).

${ }^{16}$ Archives de Tournai, Compte d'ouvrages du 20 août 1404 au 20 novembre 1404, n 2518 de l'inventaire, $2^{\mathrm{e}}$ somme des mises, fol. $5^{\mathrm{v}}$, cité par A. Cauchie, La grande procession de Tournai (Louvain, 1892), p.17. 
et Saint-Omer. Cette procession rassemblait donc les villes françaises et flamandes dans une ville située à la croisée des routes de ces deux régions, occasion à n'en pas douter d'échanges dramatiques entre les deux cultures. De même, on trouve régulièrement des mentions d'invitations entre une ville française et une ville flamande, comme lorsque Lille reçoit une invitation à participer à un concours dramatique à Gand en $1442 .^{17}$

A Saint-Omer également, les relations avec les villes flamandes, en particulier Gand et Bruxelles, sont fréquentes. Dans le compte de 1455-56, le sergent Denis de Seninghem est payé
pour ung voiage par lui fait à Gand devers notre tres redoubté seigneur Monseigneur De Charolois pour savoir et enquere de la venue par decha de mondit seigneur, aussi veoir comment ceulx dudit lieu de Gand le recepveroient, quelz mistères ou choses ilz feroient à sa venue et quel chemin il tenroit [. . .] dont ledit Denis à sen retour a fait rellacion au long à mesdisseigneurs, et si leur a rapporté et baillié les mistères par escript que lesdits de Gand firent et démonstrèrent à ladite venue de mondit seigneur. ${ }^{18}$

On voit que les relations entre les deux villes sont cordiales au point que Gand confie le texte des mystères à Saint-Omer. De même, on a tout lieu de penser que SaintOmer participe aux concours organisés par Gand, puisque dans le compte de 153839, 'Cornille Setz, messager de la ville de Gand, reçoit 30 sous pour avoir apporté la carte contenant les pris des jeux de Rhétoricque qui se debvoient faire en ladite ville de Gand. ${ }^{19}$ Il en va de même pour Bruxelles, puisque dans le compte de 1443-44, 'Jehan Descamps, dit Wastelet, barbier' reçoit 25 sous 'au retour des esbatemens fais à Bruxelles auquel lieu il a esté pluseurs journées à ses dépens, jouant jeux de personnaiges en rime'. ${ }^{20}$

Plus singulier, on trouve dans les registres de compte de Saint-Omer deux mentions de représentations de pièces en flamand, seule trace conservée à ma connaissance de ce type de représentation dans la région: au compte de 1475-76, on donne 4 sous à 'Gherard Staes pour ung jeu de sotye qu'il a joué en flamencq en l'an de ce compte le jour des ingnocens, pour les despens de ung chariot à chevaulx qui ledit jour le menèrent avant les rues comme il est accoustumé',21 et à celui de 1497-98, on paye 16 sous

Jehan de Dohen, Pierre Stelman, Jehan Riez, Gilles de le Croix et autres jusques au nombre de dix ou douze personnaiges ayans joué en flamencq l'istoire d'un duc de

${ }^{17}$ A. La Fons de Mélicocq, cité par Britte-Ashford, 'Le théâtre populaire en Bourgogne', p. 403.

${ }^{18}$ Cité par J. de Pas, 'Mystères et jeux scéniques à Saint-Omer aux $\mathrm{XV}^{\mathrm{e}}$ et $\mathrm{XVI}{ }^{\mathrm{e}}$ siècles', Mémoires de la Société des Antiquaires de la Morinie, 31 (1913), 345-77 (p. 360).

${ }^{19}$ De Pas, 'Mystères et jeux scéniques à Saint-Omer', p. 363.

${ }^{20}$ De Pas, 'Mystères et jeux scéniques à Saint-Omer', p. 361.

${ }^{21}$ De Pas, 'Mystères et jeux scéniques à Saint-Omer', p. 375. 
Millam nommé Archises, sur un hourt fait au devant le scelle le XXII ${ }^{\mathrm{e}}$ jour de ce présent mois d'aoust que ordonné fu faire esbatemens et jeus pour la paix d'entre le Roy et notre très redoubté seigneur Monseigneur l'Archiduc. ${ }^{22}$

La question de la langue ne fait donc pas obstacle à ce que des villes se rencontrent, puisqu'en retour, Wim Hüskens montre que les villes flamandes organisent elles aussi des représentations en français:

In cities as far north as Ghent and Bruges there are archival records related to dramatic performances in languages other than Flemish. In 1496, for example, on the night of the town's annual Holy Blood Procession (3 May), the Bruges authorities, on behalf of the religious order of the Carmelites, offered a prize for the best and most enjoyable esbatement (presumably a farce), 'in wat talen het zij' [in whatever language it be]. A year later, during an archers' festival on 20 May 1497, the best esbatement 'in walscher talen' [in the French language] was given a special prize. ${ }^{23}$

On peut donc penser qu'en vertu de ces échanges dramatiques, les gravures et peintures des peintres flamands montrant des représentations dramatiques peuvent avoir valeur de document également pour les villes du nord de la France, pour autant que ces représentations soient figurées de manière réaliste.

Ceci étant établi, j'aimerais me pencher sur le cas d'une image en particulier. Il s'agit d'une gravure de Bruegel, la Kermesse de la saint Georges, assez similaire à la Kermesse d'Hoboken du même Bruegel, mais qui présente quelques détails intéressants. ${ }^{24}$ En haut au centre on peut voir l'habituelle estrade sur laquelle on représente une farce, installée devant une taverne sur de hauts tréteaux à hauteur du regard, mais on peut observer également à gauche de l'image une intéressante dramatisation de l'histoire de saint Georges combattant le dragon (et l'on peut noter la division symbolique mise en place par la gravure: à la cour de la taverne appartient la farce, à l'espace de l'église appartient la représentation de la vie de saint). ${ }^{25}$ Cette fois, on joue à même le sol la scène du chevalier qui combat le dragon, ici fait probablement de bois, posé sur des roulettes et actionné par un comparse, sous les yeux de deux autres acteurs jouant sans doute la jeune fille promise au monstre et son père (le personnage féminin est sensiblement plus petit que le personnage masculin). Dans cet espace de jeu, il est frappant de constater qu'il n'y a pas de séparation entre les acteurs et le public. Si un personnage tente de retenir deux spectateurs de l'autre côté du muret de l'église en étendant les bras, en revanche d'autres spectateurs, derrière le

${ }^{22}$ De Pas, 'Mystères et jeux scéniques à Saint-Omer', p. 375.

${ }^{23}$ W. Hüsken, 'Politics and Drama: The City of Bruges as Organizer of Drama Festivals', dans The Stage as Mirror: Civic Theatre in Late Medieval Europe, éd. par Alan E. Knight (Cambridge: Brewer, 1997), pp. 165-87 (pp. 165-66).

${ }^{24} \mathrm{Je}$ m'appuie pour cette étude sur la version de la Kermesse de la Saint Georges gravée et publiée par Jerome Cock, conservée au British Museum (date inconnue), reproduite en fin d'article.

${ }^{25}$ Je remercie Jelle Koopmans d'avoir attiré mon attention sur ce point. 
chevalier, assistent au spectacle sans qu'aucune barrière ne soit matérialisée entre eux et les acteurs. Cette scène est particulièrement remarquable: elle montre qu'à côté de la représentation traditionnelle qui consiste à montrer le jeu dramatique sur une scène qui sépare nettement le public des acteurs, il peut exister un autre type de représentation dramatique plus proche du mime, des tableaux vivants et des scènes de fête des Fous ou des Innocents, qui n'est pas jouée sur une scène ou un chariot, mais au niveau du public, de manière en fin de compte beaucoup plus visible pour les spectateurs qui n'ont pas à se tordre le cou pour suivre l'action. Il semble donc dans ce cas que si, comme le souligne Michel Rousse concernant le théâtre de la farce, 'l'espace scénique est un système clos qui se fonde au départ sur le principe d'exclusion du jeu de tous ceux qui n'ont pas de place sur cet espace privilégié', ${ }^{26}$ il n'est pas forcément nécessaire de créer une séparation physique pour marquer la démarcation symbolique entre jeu et vie réelle, et que les spectateurs sont capables d'appréhender cette distance sans qu'elle soit matérialisée dans l'espace de jeu.

Cependant, la plupart des gravures de ce type ne nous renseignent que sur les kermesses villageoises ou, faudrait-il plutôt dire, les fêtes annuelles (qui ont également lieu dans les villes) qui sont l'occasion de toutes sortes de réjouissances, dont des représentations théâtrales. Mais elles nous présentent l'image d'un public indifférencié, dont il est impossible de déterminer l'origine sociale.

Or il apparaît, au regard des témoignages écrits que l'on peut trouver dans différentes villes du Nord, qu'il n'y a pas un mais des publics pour les différents types de spectacles théâtraux qu'on a pu constater. On peut discerner au moins trois facteurs de différentiation des publics, qui entrainent une séparation des spectateurs en groupes à partir du niveau de richesse et d'éducation. Le premier repose sur le degré de compréhension que les spectateurs peuvent avoir d'une pièce, et qui divise, au sein d'un même public, les spectateurs selon ce qu'ils peuvent comprendre. Les deux autres concernent la difficulté d'accès de certaines parties du public à certains spectacles, soit pour des raisons matérielles (spectacles trop chers pour le public populaire ou joués dans un espace clos qui exclut une partie de ce public), soit pour des raisons idéologiques (le Magistrat restreignant l'accès à certaines pièces parce qu'il les trouve dangereuses pour une partie du public).

Un premier motif de séparation entre différents groupes au sein d'un même public réside dans le degré de compréhension que les spectateurs peuvent avoir d'un même spectacle. En effet, cette différence dans la compréhension peut avoir une incidence sur les réactions des spectateurs, ainsi que sur leur éventuel désintérêt pour un type de représentation qu'ils ne comprendraient pas. On peut reprendre les différents types de représentation pour tenter de cerner les réactions du public. Jusqu'au milieu

${ }^{26}$ M. Rousse 'L'Espace scénique des farces', dans Le théâtre au moyen âge: Actes du deuxième colloque de la Société Internationale pour l'étude du Théâtre Médiéval, 11-14 juillet, 1977, éd. par G. Muller (Montréal: L’Aurore/Univers, 1981), pp. 137-46 (p. 144). 
$\mathrm{du} \mathrm{XVI}^{\mathrm{e}}$ siècle, l'attitude du public lors de la représentation des mystères ne donne pas matière à commentaire, et l'on ne sait que peu de choses, sinon que les représentations suscitaient un grand engouement. Tous viennent assister aux grands mystères, au point, comme à Amiens en 1445, que l'échevinage prescrive des mesures spéciales pour contenir la foule et qu'on décide de fermer toutes les portes de la ville sauf deux et d'instaurer un tour de garde au beffroi pour surveiller la ville vidée de ses habitants, puisque la représentation aura lieu à l'extérieur des murs. ${ }^{27}$ De même, à Mons en 1501, la représentation connaît un grand succès, puisqu'on doit de même appointer des guetteurs pour surveiller la ville. ${ }^{28}$ On n'en sait pas plus sur les réactions du public, sinon que ce dernier n'est pas toujours silencieux:

Sur les réactions sentimentales et esthétiques de cette immense foule de plusieurs milliers de personnes, nos textes ne nous apprennent rien. Qu'elle ait été, surtout les personnes debout, parfois inattentive et bruyante, c'est ce qu'atteste l'expression de silete, appliquée à l'intermède musical de buccines et trompettes, destiné à lui imposer silence pendant le temps nécessaire à un acteur pour se déplacer d'un lieu à un autre et la préparer à ouïr une tirade importante. Le ProloguEUR, aussi, avant chaque Journée ou demi-Journée, multiplie les invitations au calme. ${ }^{29}$

Cependant, il n'existe pas de témoignage permettant de croire que le public n'est pas attentif et respectueux de la teneur religieuse de la pièce. Dans la deuxième moitié du $\mathrm{XVI}^{\mathrm{e}}$ siècle, il n'y a en effet pas eu d'interdiction généralisée des mystères dans le nord de la France comme ce fut le cas à Paris en 1548. Bien que la dernière grande Passion soit jouée à Valenciennes en 1547, dans la mesure où l'on ne représente plus ensuite de pièce d'une telle ampleur tant du point de vue du texte que de celui de la mise en scène, on trouve cependant des mentions de mystères jusqu'à la fin $\mathrm{du} \mathrm{XVI}{ }^{\mathrm{e}}$ siècle. Certes, des doutes émergent concernant le bien-fondé de ce type de pièces ou du moins de la possibilité de laisser des troupes profanes et probablement professionnelles jouer des pièces à caractère religieux. Ainsi à Amiens en 1560, les délibérations du Magistrat reproduisent le débat concernant la légitimité de laisser jouer un certain Jacques Macron:

Veue la requeste de Jacques Macron et des compaignons de jouer l'Apocalypse, sire Adrien Villain a esté d'advis que avant d'accorder ladite permission, ils doivent montrer les jeux qu'ilz entendent jouer pour les communiquer aux docteurs de l'eglise, attendu que, par la Saincte Escripture, il est deffendu que telle manière de gens jouent publicquement la parolle de Dieu.

${ }^{27}$ Voir A. Dubois, Les Mystères à Amiens dans les $X V^{e}$ et XVI siècles (Amiens: Typographie du Mémorial, 1878), p. 9, et A. de Calonne, Histoire de la ville d'Amiens, 3 vols (Amiens: Piteux Frères, 1899), I, 344-46, qui cite le registre aux délibérations échevinales d'Amiens, BB 18, fol. 140.

${ }^{28}$ G. Cohen, 'Une grande représentation en juillet 1501', dans Etudes d'Histoire du théâtre en France au Moyen Âge et à la Renaissance (Paris: Gallimard, 1956), pp. 231-44 (p. 243).

${ }^{29}$ Cohen, 'Une grande représentation en juillet 1501', p. 243. 
La troupe reçoit permission de jouer, 'octroyee pour huict jours, attendu que les joeux qu'ils voellent jouer sont imprimez avec privilège du roy'. ${ }^{30} \mathrm{Il}$ me semble que ce que l'on met en doute ici, c'est la capacité d'une ' telle manière de gens' à jouer correctement un mystère et, partant, la pièce qu'ils présentent apparaît elle aussi suspecte. En revanche, à Saint-Quentin, en 1567, on laisse jouer les confrères de l'Hôpital Saint-Jacques qui demandent la permission de représenter l'Histoire de saint Jacques, comme ils le font chaque année, et à Amiens, en 1597, les grandsvicaires jouent encore le Mystère de saint Joseph. ${ }^{31}$ On peut donc penser que, contrairement à ce qui se passe à Paris, où, vers le milieu du XVI $\mathrm{X}^{\mathrm{e}}$ siècle, 'souvent advient derision et clameur publicque dedans le theatre meme, tellement qu'au lieu de tourner a edification, leur jeu tourne a scandale et derision, ${ }^{32}$ le public montre une réserve suffisante pour que la représentation de pièces religieuses continue, sinon systématiquement, du moins de façon assez régulière.

Nous ne possédons pas d'éléments nous permettant de nous faire une idée de la compréhension et de l'attitude du public lors de représentations de farces, mais l'on peut penser que pour ce type de pièce, la question de la compréhension du public se pose moins. En revanche, le cas des moralités et scènes allégoriques ou bibliques jouées lors de processions ou d'entrées royales pose problème. En effet, l'on peut dire avec Gustave Cohen que, dans le cas des mystères, 'la plupart des thèmes dramatiques en étaient connus par le catéchisme ou par l'image', ${ }^{33} \mathrm{~d}$ 'autant plus que les mystères représentés sont le plus souvent la Passion de Notre-Seigneur Jésus-Christ, sa Vengeance, ou bien la vie d'un saint patron local (saint Quentin à Abbeville, sainte Waudru à Mons, saint Firmin à Amiens, etc.), que chacun connaît au moins dans les grandes lignes. En cela, les tableaux présentés parfois lors de processions font appel aux mêmes références puisqu'il s'agit là encore de toucher l'ensemble des spectateurs dans leur piété. Ainsi à Béthune, lors de la procession du Saint Sacrement, les sujets des tableaux vivants reprennent des scènes de la Passion, mimées, organisés par les différents corps de métiers de la ville: les tanneurs et cordonniers jouent la Nativité à cinq personnages et les Trois Rois à six personnages, les marchands de blé l'Entrée à Jérusalem à seize personnes, les drapiers la Résurrection de Lazare à huit personnages, etc. ${ }^{34}$

${ }^{30}$ Cité par G. A. Runnalls, Les Mystères dans les provinces françaises (Paris: Champion, 2003), p. 263.

${ }^{31}$ R. Lebègue, 'L'évolution du théâtre dans les provinces du Nord', dans La Renaissance dans les provinces $d u$ Nord (Paris: CNRS, 1956), pp. 117-26 (p. 124).

32 Arrêt du Parlement de Paris cité par L. Petit de Julleville dans Les Mystères, 2 vols (Paris: Cerf, 1880), I, 423.

${ }^{33}$ Cohen, 'Une grande représentation en juillet 1501', p. 243.

${ }^{34}$ Registres de comptes de Béthune cités par A. De La Fons-Mélicocq, 'Drames du XVI ${ }^{\mathrm{e}}$ siècle', Annales archéologiques de Didron, 8 (1848), 269-70. 
En revanche, les tableaux présentés lors des entrées royales font appel à des connaissances plus approfondies de la Bible, de l'Histoire, voire de la mythologie au $\mathrm{XVI}^{\mathrm{e}}$ siècle, car ils s'adressent avant tout au prince qui fait son entrée dans la ville, et à sa suite. De fait, si tous les habitants de la ville sont certainement présents pour assister à ce spectacle extraordinaire qu'est l'arrivée du Roi de France ou du Duc de Bourgogne dans leur ville, l'intérêt de tous les spectateurs n'est pas forcément uniforme. Tout d'abord, le dispositif scénique utilisé lors des entrées royales est mis en place avant tout pour s'offrir au regard du prince: ainsi que le souligne Joël Blanchard,

l'existence de dispositifs mobiles, chariots par exemple, dans les entrées, obéit à une finalité différente dans la mesure où les chariots déambulant dans les rues sont vus par tous, alors que la fixité des échafauds les destine prioritairement au regard du roi, qui s'arrête devant chacun lors de sa progression. ${ }^{35}$

Dans le Nord, la majorité des récits d'entrées royales que nous avons conservés désigne précisément la scène, soit comme hourd, soit comme échafaud, à Abbeville, ${ }^{36}$ Douai, ${ }^{37}$ Saint-Omer, ${ }^{38}$ Tournai, ${ }^{39}$ etc. Alors que l'expression qui désigne les acteurs jouant de courtes pièces devant le public de la ville uniquement pour une fête locale ou lors d'une procession est bien 'joueurs sur chars'. Ainsi, le règlement de $1453 \mathrm{du}$ concours dramatique qui se déroule pendant la procession de Lille précise que les acteurs joueront sur des scènes mobiles, 'sur cars, carettes, esclans ou escaffaulx portatifs'. ${ }^{40}$ La différence entre le statut des deux types de scènes apparaît clairement dans les registres de compte d'Amiens, à l'occasion de l'entrée de Marguerite d'Autriche en 1483: on paie dix livres à plusieurs acteurs 'pour avoir joué sur hourt en divers lieux, du long de la cauchie, à l'entrée de Madame la Dauphine, dix histoires montées par personnages', ${ }^{41}$ tandis que l'on paye à Pierre Dury, Pasquier de Bettembos et autres soixante sous 'pour avoir joué le lendemain du jour de l'entrée faite par Madame la Doffine en la dicte ville d'Amiens, ung jue de personnages, sur ung chariot, touchant le trône de Salomon et de l'anchienne hystoire dont jadis vint

35 J. Blanchard, 'Le spectacle du rite: les entrées royales', Revue historique, 308 (2003), 475-518 (p. 481).

${ }^{36}$ Ledieu, 'Première entrée de Charles VIII', p. 56.

${ }^{37}$ H.-R., Duthilloeul, 'Entrée de Charles-Quint à Douai', Archives Historiques et Littéraires du Nord de la France et du Midi de la Belgique, 3e série, 6 (1857), 252-67 (p. 256).

${ }^{38} \mathrm{~J}$. de Pas, 'Entrée et réception de souverains et gouverneurs d'Artois à Saint-Omer $\left(\mathrm{Xv}^{\mathrm{e}}\right.$, $\mathrm{XVI}^{\mathrm{e}}$ et $\mathrm{XVII}{ }^{\mathrm{e}}$ siècles)', Bulletin de la Société des Antiquaires de la Morinie, 12 (1907-11), 66120 (p. 73).

${ }^{39}$ G. Moreau, Le journal d'un bourgeois de Tournai: le second livre des chroniques de Pasquier de la Barre (1500-1565) (Bruxelles: Palais des académies, 1975), p. 338.

${ }^{40}$ A. E. Knight, Les mystères de la Procession de Lille, t. I: Le Pentateuque (Genève: Droz, 2001), p. 53.

${ }^{41}$ Registre de comptes d'Amiens cité par Dubois, Les Mystères à Amiens, p. 16. 
Franchion et la noble maison de France'. ${ }^{42}$ Que recouvre cette différence de termes? On peut penser tout d'abord que les hourds ou échafauds des entrées sont plus richement décorés et offrent des histoires plus complexes, notamment du fait que la scène est plus grande, et peut donc permettre à plus de personnages de s'y trouver simultanément: lors de l'entrée de Charles Quint à Douai en 1516, les scènes mesurent de soixante à plus de quatre-vingt-dix pieds de long, ${ }^{43}$ soit entre dix-huit et trente mètres. Faut-il penser alors que les chars seraient plus bas et donc permettraient à tous d'assister à la représentation, tandis que ce qui est montré sur le hourd ne serait visible que pour le prince, sa suite et les notables montés à cheval? Il semble aussi que l'on ne joue pas le même type de pièce sur un char ou sur un hourd: y aurait-il donc ainsi une différence à la fois matérielle et de contenu qui serait faite entre les spectacles spécifiquement destinés au prince et ceux offerts à tous? L'absence de plus amples descriptions (notamment de la teneur des pièces jouées le soir et le lendemain de l'entrée proprement dite, ainsi que de la question cruciale de la hauteur de la scène) ne permet pas de trancher, mais cette hypothèse est vraisemblable.

D'autre part, les histoires et les allégories présentées lors des entrées sont souvent complexes, et font appel à une culture biblique et historique qui n'est plus celle du commun des spectateurs. Pasquier de la Barre décrit dans son Journal les tableaux vivants représentés lors de l'entrée de Philippe II à Tournai en 1549: l'un d'eux montre d'une part comment David âgé fait couronner son fils Salomon de son vivant, et de l'autre la 'comparation': 'comment Charles [Quint], empereur, ordonnoit son filz, prince d'Espaingnes, roy, en son vivant. ${ }^{44}$ C'est là la lecture de Pasquier de la Barre, mais le tableau ne semble pas être aussi explicite. En effet, il est surmonté de deux quatrains expliquant la scène, l'un en latin, et l'autre en français:

Providus ut David divina mente propheta
Ante obitum regem te Salomona creat,
Haud aliter Cesar nulli virtute secundus
In proprio natum colocat imperio.
Comme David vray prophete autenticque,
Ains son trespas, Salomon laissa roy.
Ainsy Cesar, son chier filz magnificque,
Fait couronner en triumphant arroy.

Et Pasquier d'ajouter: 'Et pour mieulx donner l'histoire à entendre au commun populaire, estoient faicts deux aultres dictiers', ${ }^{46}$ qui sont eux beaucoup plus longs et explicites sur l'identité de César. Il est intéressant de voir que Pasquier de la Barre

\footnotetext{
${ }^{42}$ Dubois, Les Mystères à Amiens, p. 17.

${ }^{43}$ Duthilloeul, 'Entrée de Charles-Quint à Douai', pp. 256-60.

${ }^{44}$ Moreau, Le journal d'un bourgeois de Tournai, p. 348.

${ }^{45}$ Moreau, Le journal d'un bourgeois de Tournai, pp. 348-49.

${ }^{46}$ Moreau, Le journal d'un bourgeois de Tournai, p. 349.
} 
interprète comme une attention du Magistrat envers le public populaire la présence de ces poèmes explicatifs. Cependant, y avait-il un grand nombre de gens capables de lire ces poèmes parmi ce public? A moins que ces derniers n'aient été lus à voix haute par un des acteurs sur scène ou un commentateur à côté de cette dernière, ce que Pasquier ne précise pas? Ou bien ne seraient-ils pas plutôt destinés au Prince luimême, pour s'assurer que ce dernier ait bien compris le tableau, ce que s'interdirait de suggérer Pasquier de la Barre? Il semblerait en effet, que les histoires et allégories jouées lors d'entrées royales n'aient pas toujours été très facilement déchiffrables: Anne-Marie Lecocq montre comment Froissart s'est mépris sur le sens d'allégories présentées pour l'entrée d'Isabeau de Bavière à Paris en 1389:

Si ces gens-là (les gens de savoir), n'étant pas dans le secret du programme, s'y perdent, de combien de Parisiens ou de Rouennais le message politique pouvait-il être compris? Et combien de fois est-il arrivé au roi lui-même (qui n'est pas un lettré) de ne rien comprendre à tel spectacle de rue lors d'une entrée? ${ }^{47}$

Le fait est que, plus l'on avance dans la période, plus les tableaux présentés et les références en jeu se complexifient, puisque l'on recherche plus volontiers la nouveauté et que l'on est donc amené à recourir à des comparaisons de plus en plus ingénieuses et originales. Les organisateurs des entrées en sont sans doute conscients, qui entourent de plus en plus les tableaux vivants d'explication, à mesure que l'on avance dans le $\mathrm{XVI}^{\mathrm{e}}$ siècle. Ainsi, pour l'entrée à Abbeville en 1531 d'Eléonore Habsbourg qui vient d'épouser François $I^{\mathrm{er}}$, on joue d'abord un dialogue à l'entrée de la ville entre des allégories représentant le roi, la reine et Abbeville et qui donnent l'explication des tableaux vivants placés sur le parcours de la reine à l'intérieur de la cité. ${ }^{48} \mathrm{Il}$ ne semble pas, cependant, que le public populaire se désintéresse d'allégories qu'il ne comprendrait pas: aucun texte ne mentionne une quelconque désaffection pour les entrées royales et même si l'on comprend que ces relations d'entrées, la plupart du temps de nature officielles, ont tout intérêt à ne pas mentionner ce point, il serait étonnant que le public boude ces entrées, qui sont pour lui l'occasion de voir de près des personnages importants, voire le Roi de France ou le Duc de Bourgogne en personne.

On voit donc que certaines représentations dramatiques peuvent poser problème au moins à une partie du public, et contribuer à le fractionner en groupes qui voient et

${ }^{47}$ A.-M. Lecoq, 'La symbolique de l'État: Les images de la monarchie des premiers Valois à Louis XIV', dans Les Lieux de mémoire, t. II: La nation, vol. 2, Le territoire, l'Etat, le patrimoine, dir. par Pierre Nora (Paris: Gallimard, 1986), pp. 145-92 (p. 150), citée par Blanchard, 'Le spectacle du rite', p. 493.

${ }^{48}$ Voir A. Ledieu, 'Entrée de la reine Éléonore d'Autriche à Abbeville le 19 décembre 1531', dans Mélanges d'histoire locale, lectures faites à la société d'émulation d'Abbeville de 1895 à 1900 (Paris: E. Dumont, 1901), pp. 385-92, et mon article 'Le roi et son double, a Royal Entry to Late-Medieval Abbeville', dans Actes de la journée d'étude internationale de Louvain, 'Communication in the Late Medieval Town', tenue le 14 novembre 2003 (à paraître). 
comprennent plus ou moins bien les pièces. L'accès aux pièces pour certains de ces groupes ne se pose pas seulement lors des entrées royales, et il me semble qu'on peut non seulement voir une différenciation entre ces groupes non seulement du point de vue du degré d'instruction, mais également du point de vue du niveau des ressources. En effet, la question du prix des entrées d'une pièce — mystère mais également farce ou moralité jouées dans un espace clos tel qu'une taverne - est loin d'être close. La plupart du temps, les informations manquent pour tenter de déterminer si le prix demandé était un obstacle à la venue des parties les plus pauvres du public. On peut penser que tel n'est pas le cas pour les représentations de farces, mais il est difficile d'en savoir plus pour les mystères, et l'on peut se demander dans quelle mesure une pièce telle que la Passion de Valenciennes de 1547 peut toucher un public large, alors qu'elle est représentée en vingt-cinq journées. Si les six deniers réclamés comme droit d'entrée ne sont pas un coût insurmontable quand on n'assiste qu'à un petit nombre de journées, il est vraisemblable que suivre l'ensemble des vingt-cinq journées représente un coût trop important en temps et en argent pour le public populaire, qui sélectionne alors les représentations en fonction des moments programmés chaque jour, selon l'importance des épisodes représentés et son goût personnel. ${ }^{49} \mathrm{Je}$ passe donc rapidement sur cette question, qui mériterait pourtant qu'on s'y attarde, pour en venir à une autre limitation à l'accès aux pièces en fonction des ressources. On remarque que les archives mentionnent parfois des représentations dans des espaces clos, pour un petit nombre de personnes. Il peut s'agir d'une représentation devant le duc de Bourgogne, qui fait alors jouer des pièces pour son plaisir, là où il réside (et dans ce cas, on retrouve les mentions de ces pièces dans les registres de comptes de la maison de Bourgogne). Ainsi, à Arras en 1435:

Jehannin de Lalle, Petit Jehan, Philippe de Douay, Willot de la Rue et Jehannet le Jay, joueurs de farses, demourans à Cambray [reçoivent 14 livres] pour une fois, quant ylz sont nagaires, venu dudit Cambray à Arras, jouer devant Monseigneur pluiseurs jeux de farses en personnaiges et moralitez, à la feste de Messire Helbin Hambaut, abbé des fols de l'ostel de mon dit Seigneur. ${ }^{50}$

De même, à Amiens, le 13 octobre 1476, Miquiel Roye, sans doute un notable aisé, fait représenter un 'mystère' le jour de son mariage. ${ }^{51}$ Par ces deux exemples, on voit qu'à côté des représentations organisées par la ville, il existe des représentations à titre privé, payées par ceux qui peuvent se les offrir et les faire représenter chez eux

${ }^{49}$ Voir E. Konigson, La Représentation d'un mystère de la Passion à Valenciennes en 1547 (Paris: Éditions du CNRS, 1969).

${ }^{50}$ Cité par A. De la Fons Mélicocq, 'Les Rois de la Fève, les fous en titre d'office et de la chapelle, les joueurs de farces et les mommeurs de l'hôtel de Philippe-le-Bon, duc de Bourgogne', Messagier des Sciences historiques, des arts et de la bibliographie de Belgique, 1857, pp. 393-400 (p. 395).

${ }^{51}$ Cité par G. Lecocq, Histoire du théâtre en Picardie depuis son origine jusqu'à la fin $d u$ $X V I^{e}$ siècle (Paris: H. Menu, 1880), p. 48. 
devant un public choisi, bien que la frontière entre la sphère privée et la sphère publique ne soit pas toujours clairement délimitée, par exemple quand le Magistrat participe à la dépense d'une représentation devant le duc de Bourgogne: on peut en voir un exemple dans le compte de 1438-39, où l'on donne pour 63 sous de vin aux gens du duc de Bourgogne qui ont 'fait l'esbatement de l'abbé de Joieuse Folie par devant monseigneur le duc'. 52

D'autre part, à Saint-Omer, le compte de 1436-37 attribue

à certaines personnes jueurs de personnages qui ont jué et monstré à l'ostel de le Couronne ung jeu de mistère de le paix qui a esté fait à Arras entre le Roy de France et Monseigneur le duc de Bourgogne, auquel jeu furent monseigneur le bailly de ceste ville, son lieutenant, pluseurs de ces messrs maieurs et eschevins de l'une année et de l'autre, pour ce à eulx donné $[\ldots] 4$ sols. ${ }^{53}$

Dans ce cas précis, il semble que la pièce jouée soit la Moralité de Povre Commun composée par Michault Taillevent et représentée à Arras en 1435 lors des négociations de paix entre le duc de Bourgogne et le roi de France. Selon J. Watkins, la pièce aurait pu être jouée en public à Arras. ${ }^{54}$ Or à Saint-Omer, elle n'est jouée que devant un petit nombre de personnes: le bailli, le maire et une partie des échevins. On peut se demander pourquoi le Magistrat décide de ne faire représenter la pièce que devant ses membres, plutôt que de la faire jouer devant tous sur une place publique. Est-ce parce que l'événement est déjà loin dans les mémoires, et n'intéressera plus un large public, mais que les échevins souhaitent tout de même le voir à titre privé en utilisant cependant des fonds publics? La séparation entre sphère privée et sphère publique n'est pas toujours nette, ainsi qu'on l'a vu plus haut, et comme le montre le fait que, lors de la représentation de mystères, il ne soit pas rare que le maire et les échevins dînent aux frais de la ville (au titre de 'despens de bouche fais par nosseigneurs maieur et eschevins pour le fait et honneur de la ville', du nom d'une des rubriques des registres de comptes de Saint-Omer). On trouve dans cette ville d'autres mentions qui attestent que des pièces ont été jouées devant le Magistrat aux frais de la ville. Ainsi, en 1442, un joueur d'ébattements joue 'devant messires maieur et eschevins le fait de l'armée du Roi nostre sire et les emprinses qu'il avoit faites ou pais bordelois'. ${ }^{55}$ Il ne semble pas que ces représentations aient pour but de vérifier le contenu de la pièce avant de la représenter en public. En effet, ces représentations face à un public choisi ne sont jamais accompagnées, d'après les archives, de délibérations concernant l'autorisation ou l'interdiction d'une représentation publique, ni suivies de représentations des mêmes

\footnotetext{
${ }^{52}$ Cité par De Pas, 'Mystères et jeux scéniques à Saint-Omer', p. 354.

${ }^{53}$ De Pas, 'Mystères et jeux scéniques à Saint-Omer', p. 359.

${ }^{54}$ J. Watkins, 'A Fifteenth-Century Morality Play: Michault Taillevent's Moralité de Povre Commun', French Studies, 8 (1954), 207-32 (p. 208).

${ }^{55}$ De Pas, 'Mystères et jeux scéniques à Saint-Omer', p. 355.
} 
pièces face à l'ensemble de la population de la ville. De plus, on paye les acteurs, ce qui montre qu'il s'agit là de récompenser une pièce et des acteurs qui ont procuré un divertissement, contrairement à ce qui se passe plus tard, surtout au XVI siècle.

Il apparaît donc qu'il existe dans un certain nombre de cas une séparation physique des publics, et que jouer dans un espace clos et relativement petit, la pièce d'une maison le plus souvent, signifie jouer pour un public aisé, qui ne souhaite pas se mêler au public populaire pour assister à une représentation, quand bien même cette dernière serait celle d'une farce. Il existe pourtant un type d'espace clos spécifique qui nie cette séparation en publics aisé et populaire: la taverne. De fait, on a conservé quelques témoignages de pièces jouées dans des tavernes à Amiens. En 1449, Motin et ses 'compaignons facteurs' jouent 'le jeu d'Odenguier [Audigier] dans le cellier de la Seraine [Sirène]', au Marché aux Herbes ${ }^{56}$ la même pièce est représentée par des 'joueurs de personnages' à la taverne du Cornet d'Or le 11 janvier $1473 .{ }^{57} \mathrm{Il}$ s'agit sans doute d'une mise en scène de la Chanson d'Audigier, une parodie scatologique de chanson de geste. ${ }^{58}$ On joue également deux pièces à la taverne de la Faucille (ou de Famille selon Lecocq) en 1481: Vinchenot et Rosette le 2 janvier et Peu de grain et largement eau le 27 février (sans doute deux farces, d'après le titre). ${ }^{59} \mathrm{Il}$ s'agit là évidemment d'occasions pour tous de venir assister à la pièce, et si l'on ne sait pas quelle est la composition du public des pièces jouées lors de ces occasions, on peut penser qu'il s'agit d'un public varié, la présence d'un public populaire n'excluant pas celle d'un public aisé. L'endroit ne se présente pas comme l'opposé des places publiques où l'on joue le même type de pièces, ni même comme celui des lieux où l'on joue les mystères. En effet, comme le souligne Guy Paoli, 'la relation entre la taverne et les tréteaux est scellée par le vin que l'on offre aux acteurs, qui parfois représente leur salaire', ${ }^{60}$ non seulement pour les acteurs de farces, mais aussi pour les acteurs de pièces religieuses. ${ }^{61}$

Tout change au XVI ${ }^{e}$ siècle. A ce moment-là, en effet, le fait de jouer une pièce devant un petit nombre de personnes, le plus souvent des membres du Magistrat ou des autorités ecclésiastiques, n'est plus le signe qu'on procure un divertissement à un

\footnotetext{
${ }^{56}$ Dubois, Les Mystères à Amiens, p. 11.

${ }^{57}$ Dubois, Les Mystères à Amiens, p. 14.

${ }^{58}$ Voir notamment O. Jodogne, 'Audigier et la chanson de geste, avec une édition nouvelle du poème', Le Moyen Âge, 66 (1960), 495-526.

${ }^{59}$ Jodogne, 'Audigier et la chanson de geste', p. 15, et Lecocq, Histoire du théâtre en Picardie, p. 138.
}

${ }^{60}$ G. Paoli, 'Théâtre et taverne au Moyen Âge', dans Théâtre et spectacles hier et aujourd'hui, pp. 73-82 (p. 80).

${ }^{61}$ Pour ne prendre qu'un exemple, voir Cohen, 'Une grande représentation en juillet 1501', p. 241, qui montre comment on récompense l'acteur ayant joué Dieu le Père par un don en vin, et comment les acteurs jouant Dieu le Père et Dieu le Fils fréquentent plutôt la taverne $\mathrm{Au}$ Cerf, tandis que les acteurs jouant les diables vont à la taverne de la Clef. 
public spécifique, mais qu'on soumet sa ou ses pièce(s) à l'attention d'un comité de censure. On assiste en effet, à partir de la fin $\mathrm{du} \mathrm{XV}^{\mathrm{e}}$ siècle, et à mesure que l'on avance vers la fin de la période, à une surveillance accrue du contenu des pièces de la part des échevins et des autorités religieuses. L'on continue certes à jouer des mystères dans le Nord, lorsque ceux-ci sont organisés par des bourgeois de la ville, et à favoriser les échanges dramatiques avec les villes voisines, au point même de parfois relancer l'intérêt pour une fête tombée en désuétude, comme lorsque Lille décide de remettre à l'honneur la fête du Prince de Plaisance en 1547. Cette même fête est ensuite organisée à Tournai la même année, puis à Valenciennes l'année suivante. Simon Le Boucq l'évoque en détail dans son Histoire de Valenciennes (écrite vers 1650), et la précision de ses descriptions laisse penser qu'il les tient d'une source manuscrite de l'époque de la fête. Il donne les raisons suivantes pour expliquer le renouveau de la fête de Plaisance:

De touttes anciennetées lorsque les pays florissoient en paix, plusieurs villes de ces
provinces faisoient, tous les estées, des récréations, triumphe fort considérables pour
s'entretenir avec leurs voisins en bonne et parfaicte amitiée, aiant en chaque ville où
cecy se praticquoit un Prince, lequel donnoit les ordres à tout, soit pour recepvoir ceux
des aultres villes, ou bien y aller, ainsi que le cas le requéroit, et ceste feste solemnelle
qu'on appelloit Principauté se tenoit ordinairement en temps susdite le dimanche après
l'Ascension de Notre Rédempteur. Et comme les guerres passées et les misères
survenues avoient faict mectre en oubliance tout cecy, ceux de Lille, de Tournay,
voiant le pays en tranquilité, délibérèrent de remectre sus icelle feste en l'an $1547 .^{62}$

Après avoir reçu les compagnies de bourgeois des villes environnantes, dont celles de Lille, Tournai, Ath, et même de Reims, le prince de Plaisance les invite à un dîner,

après lequel repas, ceux de villes et villaiges susdis vindrent les uns après les autres faire leurs farces et comédies devant le logis dudit Prince, lequel faisoit distribuer à chaque acteur ung cigne d'argent, et puis alloient encore jouer en divers endroits de la ville, de manière que cela dura jusques vers deux heures de nuict, le tout avec si bon ordre qu'aucune noise ou débat fut veue. ${ }^{63}$

Cependant, il s'agit là de fêtes et de représentations organisées par ceux-là mêmes qui détiennent le pouvoir dans la cité; en revanche, on interdit progressivement les fêtes de nature subversive, telle la fête des Fous ou celle des Innocents, et le Magistrat surveille de près les représentations dramatiques organisées par des confréries de quartier tandis que les autorités religieuses contrôlent celles des prêtres, qui sont de moins en moins autorisés à participer à des représentations suspectées de blasphème ou d'hérésie. Ainsi, le synode de Cambrai de 1550 renouvelle une interdiction

${ }^{62}$ Cité par F. Brassart, 'Fêtes populaires au XVI ${ }^{\mathrm{e}}$ siècle dans les villes du nord de la France, et particulièrement à Valenciennes (1547 et 1548)', Souvenirs de la Flandre-Wallonnne: recherches historiques et choix de documents relatifs à Douai et la Province, 11 (1871), 4674 (p. 55).

${ }^{63}$ Brassart, 'Fêtes populaires au XVI ${ }^{\mathrm{e}}$ siècle', p. 68. 
précédemment faite aux prêtres de jouer des pièces qui parodieraient la liturgie: 'ne se immisceant missis artificiosis'. ${ }^{64}$

De même, la représentation de pièces à caractère profane ou par des troupes d'acteurs venues de l'extérieur de la région est de plus en plus soumise à un contrôle. On peut citer deux exemples, l'un d'autorisation de jouer, l'autre d'interdiction, tous deux observés à Amiens. D'après une délibération de l'échevinage du 2 janvier 1555 ,

sur la requeste présentée audit échevinage par Anthoine Soene, enfant de Ronain en Dauphiné, et ses compagnons joueurs d'histoires, tragédies morales et farces adfin qu'il leur fust permis de jouer en ceste ville les dites moralités et farces sur icelle en advis audit eschevinage il leur a esté permis jouer en chambres moralités honnestes et non sentant aucun point d'hérésie l'espace de six jours. ${ }^{65}$

En revanche, dans le registre aux délibérations de l'échevinage de 1567, on trouve la délibération suivante:

Sur ce que Samuel Treslescat et ses compagnons joueurs ou réciteurs d'histories, tragédies, comédies, farces, etc, se sont présentées devant Messieurs et leur ont demandé l'autorisation de jouer en cette ville, suivant la permission qu'ils ont obtenue de Mgr le prince de Condé, gouverneur et lieutenant-général pour le roy en Picardie, mesdits sieurs après avoir mis l'affaire en délibération ont conclu: de ne pas permettre, quant auxdits Treslescat et ses compagnons de jouer et reciter en ceste ville, pour obvier à toutes noises et débats qui, souvent, se sont faictz en pareille assemblée; comme aussi aux maladies qu'il peut en arriver, par les chaleurs où nous sommes; attendu, encores, les éditz du roy, les arrests de la cour, la cherté des vivres, la pauvreté du menu peuple qui pourrait perdre son temps à voir ces jeux; enfin à cause des troubles et des degatz des gens de guerre, et pour plusieurs autres bonnes raisons et considérations qui ont été plus amplement déduites. ${ }^{66}$

D'après ces deux délibérations, on peut tirer un certain nombre de conclusions à propos des raisons qui poussent le Magistrat à contrôler les troupes souhaitant jouer des pièces et à n'en proposer qu'une partie au public dans son ensemble. Il s'agit d'abord de vérifier le contenu théologique de certaines pièces: dans une région qui connaît une montée du protestantisme, on surveille de très près un spectacle qui peut facilement devenir un instrument de prosélytisme. Les raisons données pour interdire une représentation dans la deuxième délibération sont plus complexes: elles relèvent d'un désir de contrôler une partie du public désormais perçu comme incapable de savoir où se trouve son intérêt, qu'il faut donc guider pour l'empêcher de 'perdre son temps', et qui est aussi considéré comme facteur de troubles pour la cité, soit par

${ }^{64}$ E. Fleury, Origines et développements de l'art théâtral dans la province ecclésiastique de Reims (Laon: A. Cortilliot, 1880), p. 93.

${ }^{65}$ A. Dubois: Récréations de nos pères aux $X V^{e}$ et $X V I^{e}$ siècles (Amiens: L. Challier, 1860), p. 11, accentué par moi.

${ }^{66}$ H. Dusevel. Les Joueurs de farces à Amiens, fragment d'une histoire de la Picardie (Amiens: Lenoel-Hérouart, 1861), p. 3, accentué par moi. 
l'agitation que pourrait causer en lui une pièce, et qui pourrait nuire à l'ordre public, soit par le simple fait que des rassemblements de foule peuvent favoriser la propagation des maladies. Il faut noter qu'il s'agit ici d'une troupe d'acteurs venus d'une autre région. Comme on l'a vu, ces étrangers à la ville sont considérés avec méfiance, et avec eux, les pièces de leur répertoire, fussent-elles autorisées par le roi. De fait, à la même époque, on accepte beaucoup plus facilement qu'un acteur local joue une pièce pour ce même public qu'on voulait par ailleurs empêcher de se rassembler. Ainsi, en 1546 à Béthune, Michel Gambon, maître de la grande école de la paroisse de Béthune, reçoit deux cannes de vin 'pour avoir rescréé le peuple et enseigné les jones enffans en ung ju moral avec farse joieuse' ${ }^{67} \mathrm{Il}$ semble donc que, si les autorités de la ville ont toujours à l'esprit la notion de public populaire qu'il faut surveiller et contrôler, la différence se situe surtout au niveau de l'origine des acteurs, leur attribuant ainsi une responsabilité dans l'encadrement de ce public: des acteurs étrangers à la région ne connaîtront pas le contexte régional et son public, et risquent par-là même de susciter des réactions incontrôlées, tandis que les acteurs locaux et régionaux ne représentent pas un danger car ils savent comment canaliser les réactions de la foule, en la récréant tout en l'instruisant.

Il apparaît donc que ce public du théâtre dans le nord de la France, a priori difficile à définir, n'est pas totalement insaisissable. A partir de documents iconographiques qui nous renseignent au moins sur un certain type de représentations au XVI ${ }^{\mathrm{e}}$ siècle, on est amené à revoir le jugement selon lequel le public devrait être forcément exclu de l'espace de jeu par une séparation physique. De plus, grâce à des mentions d'archives plus nombreuses qu'on ne pouvait le penser de prime abord, on constate une différenciation des publics selon les types de pièces représentées. On s'aperçoit ainsi notamment que le public populaire est progressivement cantonné à des pièces perçues comme inoffensives et peu critiques, tandis qu'on assiste à une disparition progressive des pièces qui pouvaient éventuellement provoquer une contestation du pouvoir civil et religieux, notamment du fait de la montée du protestantisme dans la région, ainsi qu'à un repli des cités sur elles-mêmes face à la montée d'un théâtre professionnel dont les acteurs viennent de l'extérieur de la région. Plus que l'apparition de pièces nouvelles qui supplanteraient les pièces de type médiéval dans le cœur du public, il me semble que c'est cette volonté de contrôle du public populaire qui précipite le déclin des pièces qui jusqu'alors récréaient l'ensemble de la population, et qui marque la fin d'une époque.

Universiteit van Amsterdam

${ }^{67}$ Compte de l'argentier de Béthune cité par G. de Hautecloque, Les représentations dramatiques dans les collèges de l'Artois avant 1789 (Abbeville: Cabinet historique de l'Artois et de la Picardie, 1888), p. 31. 


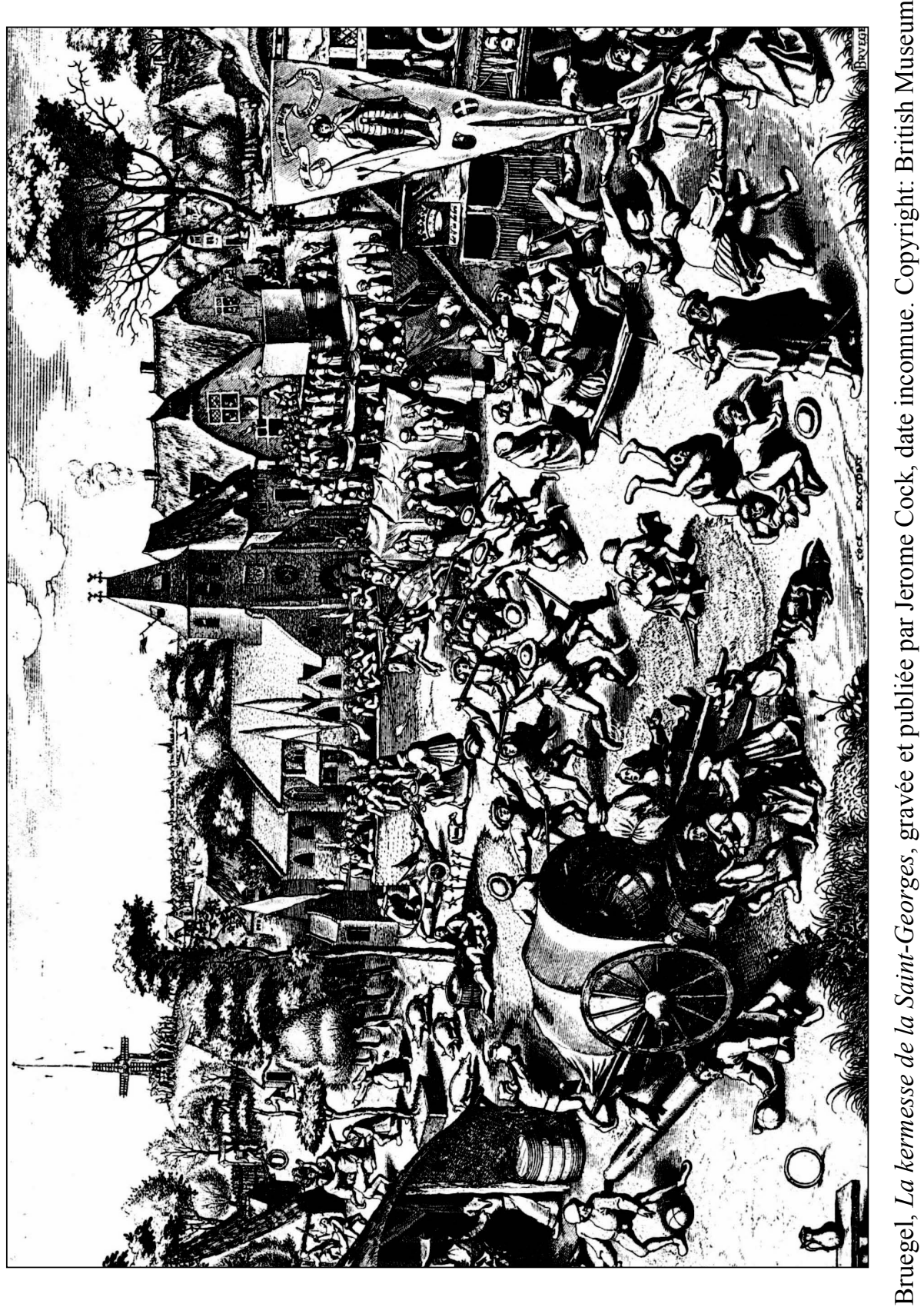

